\title{
Studying the structure of cardiovascular pathologies in patients with chronic kidney disease of the terminal stage of rural population receiving hemodialysis
}

\begin{abstract}
This article presents the results of studying the structure of the damage to the cardiovascular system in patients with terminal stage chronic kidney disease who are on programmed hemodialysis of the rural population of the Republic of Uzbekistan. Scientific research was conducted among the rural population in the Department of Nephrology and hemodialysis of the Syrdarya multidisciplinary medical center. As a result of the examination of 61 patients, it was shown that the main pathologies of CVS in this patient population are arterial hypertension, angina pectoris and chronic heart failure. It was also revealed a high comorbidity of cardiovascular pathology.
\end{abstract}

Keywords: cardiovascular, CKD, Dialysis, IHD, RRT, rural people
Volume 8 Issue 3 - 2020

\begin{abstract}
Daminov BT, I,2 Sharapov ON, ,,2 Dyagilev VA 3
'Tashkent Pediatric Medical Institute, Tashkent, Uzbekistan

${ }^{2}$ Republican specialized scientific and practical medical center for nephrology and kidney transplantation, Tashkent, Uzbekistan ${ }^{3}$ Syrdarya Regional Multidisciplinary Medical Center, Gulistan, Uzbekistan
\end{abstract}

Correspondence: Olimkhon Sharapov, Tashkent Pediatric Medical Institute, Bogishamol, 223, I00I40, Yunusabad district, Tashkent, Uzbekistan, Tel +998909722508, Fax +9987 I 2603 I 26, Emailolimkhon@gmail.com

Received: May 23, 2020 | Published: June 29, 2020
Abbreviations: CKD, chronic kidney disease; HD, hemodialysis; IHD, Ischemic heart disease; RRT, renal replacement therapy; $\mathrm{LVH}$, left ventricule hypertrophy; $\mathrm{AH}$, arterial hypertension; CVO, cardiovascular outcomes; CHF, chronic heart failure; SAS, sympathetic adrenal system; rhEPO, recombinant human erythropoietin; CBV, circulating blood volume, SHPT, secondary hyperparathyroidism.

\section{Introduction}

In a recently published joint report by the European Kidney Association (ERA-EDTA), American Society of Nephrology (ASN) and International Society of Nephrology (ISN), kidney diseases were recognized as one of the most common diseases in the world. According to this report, globally, the total number of people with chronic kidney disease (CKD), acute kidney damage (AKP) and people receiving renal replacement therapy (RRT) exceeds 850 million people, which is actually twice the estimated number of people with diabetes in around the world and 20 times higher than the number of people suffering from acquired immunodeficiency syndrome (AIDS) / human immunodeficiency virus (HIV) worldwide. CKD is currently an undeniable global public priority. ${ }^{2,3}$ Despite the fact that the prevalence of CKD and its effects on health have been studied mainly in economically developed countries, the burden of this disease is even greater in developing countries. ${ }^{4,5}$ By the end of the XX century, scientific data had accumulated, which allowed to significantly expand the idea of the relationship of renal and cardiac pathology. One of the first to establish the fact that more than $50 \%$ of deaths in patients with terminal renal failure (ESR) receiving renal replacement therapy, due to cardiovascular (SS) causes. ${ }^{6,7}$ This fact is especially revealing by the example of a population of young patients with ESRD, in which the mortality rate from CVD is 300 times higher than in the control group of the same age with normal renal function.

\section{Material and methods}

In the course of a scientific study, 61 patients who were permanently living in rural areas of the Syr Darya region were examined. The study was conducted at the Department of Nephrology and Hemodialysis of the Syrdarya Regional Multidisciplinary Medical Center during 2018. $64 \%(n=39)$ of the examined patients were men and $36 \%(n=22)$ were women. The average age of the patients was $45.5 \pm 12$ years. The study included patients with a clinically established diagnosis of CKD stage V (according to NKF K / DOQI 2002) [26] in the outcome of nephropathies of various origins. Glomerular filtration rate was calculated based on the serum creatinine concentration according to the formula CKD-Epi. The main underlying diseases were chronic glomerulonephritis $(\mathrm{n}=38)$, diabetes mellitus $(\mathrm{n}=11)$, urolithiasis $(\mathrm{n}=3)$, chronic pyelonephritis $(\mathrm{n}=6)$, systemic lupus erythematosus $(n=1)$, and polycystic kidney disease $(n=2)$ (Figure 1). The duration of HD was 16 (6-96) months. Programmed hemodialysis was performed with Fresenius Medical Care 4008S devices according to the scheme 4 hours 3 times a week ( 720 hours / week). For hemodialysis, a bicarbonate solution was used as a dialysate. Criteria for exclusion from the study were the age of 18 years, residents of the urban population of the region, patients who underwent kidney transplantation, patients receiving $\mathrm{HD}$ in acute renal failure. The examination program included general clinical laboratory research. To study the state of the cardiovascular system, all patients underwent blood pressure monitoring, electrocardiography, and echocardiography.

\section{Results}

According to the results of a study of 61 patients, cardiovascular pathology was detected in 24 ( $39 \%$ of all examined). The most frequent cardiovascular pathology encountered was arterial hypertension (AH), more than $39 \%(n=24)$ of patients suffered from it. Patients with stable angina pectoris $(\mathrm{CCH})$ turned out to be much smaller - $6.55 \%$ 
$(n=4)$ of the examined. Chronic heart failure was detected in $5 \%(n=3)$ of patients. Rhythm and conduction disturbances were also observed $(n=3)$ : in 1 case $(1.6 \%)$, patients with atrial fibrillation, ventricular extrasystole and grade 1 atrioventricular block were identified. According to the data obtained, it was found that almost $77 \%$ of patients $(n=47)$ were diagnosed with renal anemia. Arterial hypertension was observed in $24(39 \%)$ patients (14 men and 10 women). Moreover, half of them - $12(19.6 \%)$ had grade 2 hypertension, $2(3.3 \%)$ patients had grade 1 hypertension, grade 3 hypertension was observed in 10 $(\mathrm{n}=16.4 \%)$ patients. In terms of sex, $\mathrm{AH}$ of the 2 nd degree in men was found twice as much as in women (Figure 2), $\mathrm{AH}$ of 1 and 3 degrees were the same in both sexes. Angina pectoris was diagnosed in $3(4.9 \%)$ men and $1(1.6 \%)$ women of the examined patients. When distributing patients according to functional classes (FC), according to the classification of the Canadian Association of Cardiologists, there was a lack of CCN FC2 and FC4 in the examined patients (Figure 2), as well as FC1 in women. In 3 patients, FC3 was diagnosed (2 men and 1 woman), in one man $\mathrm{FC} 1$ angina was diagnosed.

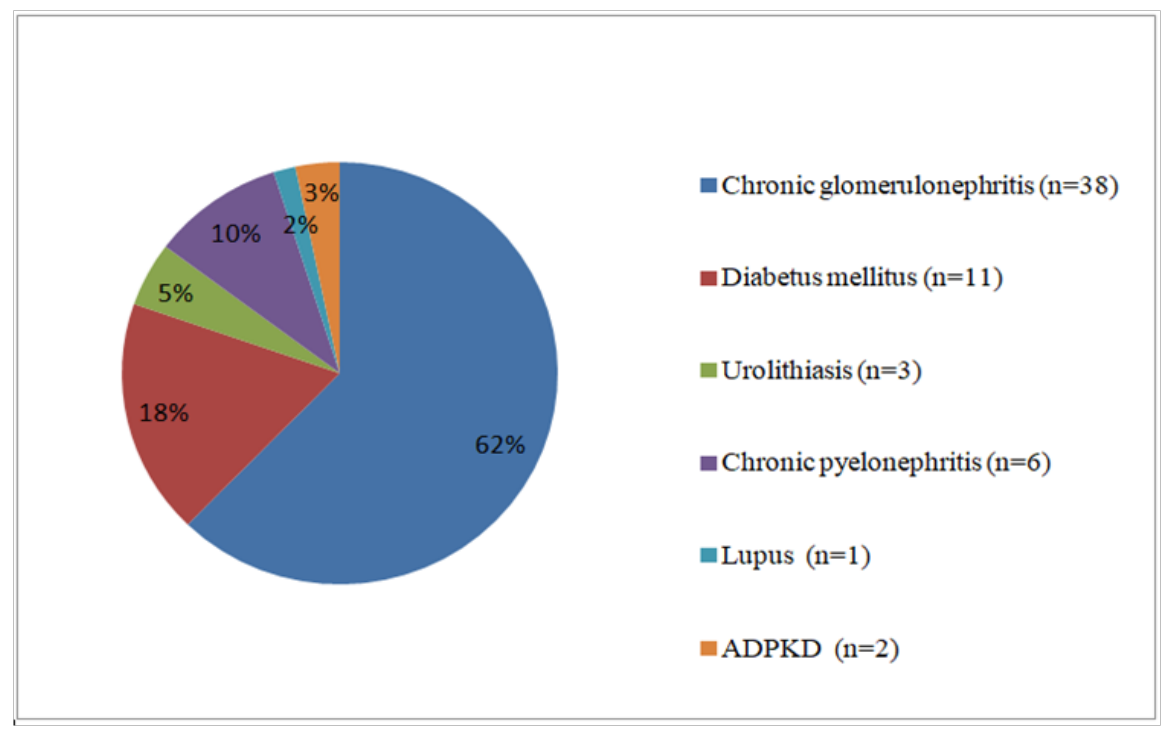

Figure I Initial nosology of the examined patients $(n=6 I)$.

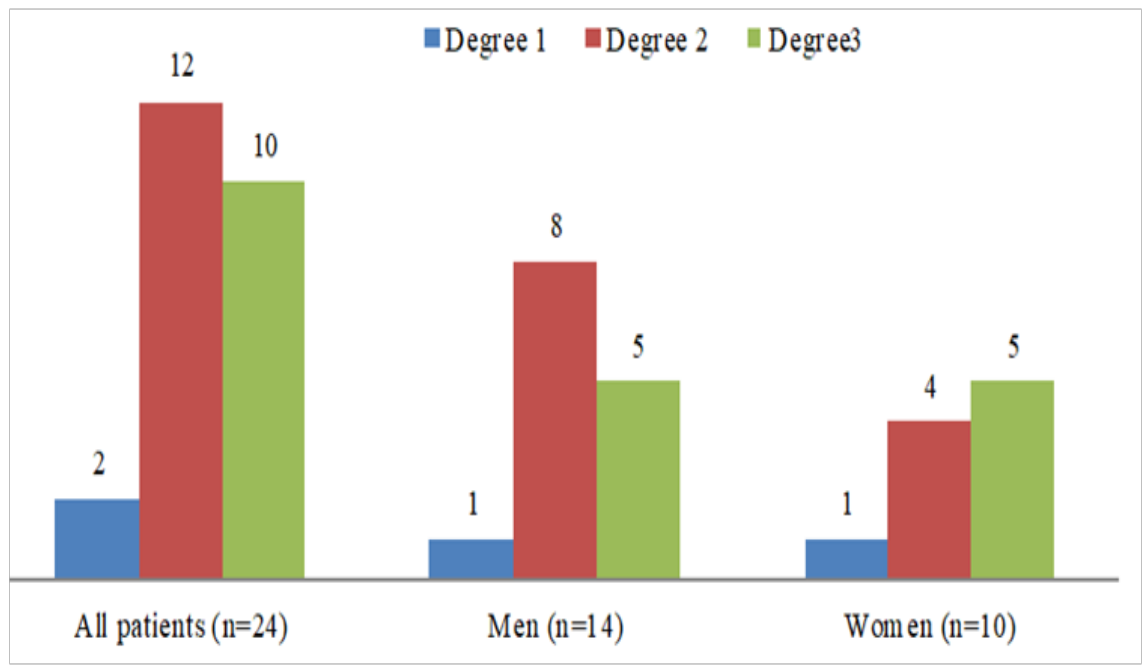

Figure 2 The prevalence of arterial hypertension in patients with CKD 5D in the rural population.

In $3(5 \%)$ examined patients, chronic heart failure was diagnosed. Patients were divided into functional classes (according to NYHA, 1964). All 3 patients had FC1 ( 2 men and 1 woman). Not a single patient was diagnosed with CHF 2-4 functional class. It should be noted that most of the examined patients showed comorbidity of cardiovascular diseases. The most frequent comorbid state was found in $\mathrm{CCH}, \mathrm{AH}$ and $\mathrm{CHF}$ in various combinations. It was found that all patients (n-24) with cardiovascular pathologies had arterial hypertension. Therefore, all 4 patients with angina pectoris also suffered from hypertension. 1 patient who was diagnosed with hypertension and angina pectoris also had chronic heart failure. The combination of $\mathrm{CHF}+\mathrm{AH}$ was observed in 2 patients. 19 (31.1\%) patients had "isolated" arterial hypertension. By gender, isolated cases of hypertension were more common in men (n-11) than in women (n-8). 


\section{Discussion and conclusion}

Cardiovascular complications occupy a leading place among pathologies in patients of the rural population of CKD on programmed hypertension. ${ }^{9,10}$ Based on the analysis of 61 patients with CKD on programmed HD, it can be assumed that the main pathologies of the cardiovascular system in patients were hypertension, angina pectoris, heart failure and various arrhythmias. According to the above results of our study, it can be assumed that the comorbid pathology of the cardiovascular system occupies a leading place in the structure of cardiovascular lesions in patients with end-stage chronic kidney disease receiving program hemodialysis and did not significantly differ by sex.

\section{Acknowledgments}

None.

\section{Conflicts of interest}

There is no any conflict of interest.

\section{Funding}

None.

\section{References}

1. Kitty J Jager, Csaba Kovesdy, Robyn Langham, et al. A single number for advocacy and communication-worldwide more than 850 million individuals have kidney diseases. Nephrology Dialysis Transplantation. 2019;34(11):1803-1805.
2. Levin A, Tonelli M, Bonventre J, et al. Global kidney health 2017 and beyond: a roadmap for closing gaps in care, research, and policy. Lancet. 2017;390:1888-1917.

3. Crews DC, Bello AK, Saadi G et al. Burden, access, and disparities in kidney disease. Kidney Int .2019;95:242-248.

4. Hill NR, Fatoba ST, Oke JL et al. Global prevalence of chronic kidney disease - a systematic review and meta-analysis. PLoS One. 2016;11:e0158765.

5. Xie Y, Bowe B, Mokdad AH et al. Analysis of the global burden of disease study highlights the global, regional, and national trends of chronic kidney disease epidemiology from 1990 to 2016. Kidney Int. 2018;94:567-581.

6. A Lindner, B Charra, D J Sherrard, et al. Accelerated atherosclerosis in prolonged maintenance hemodialysis. N Eng J Med. 1974;290:697701.

7. Simon Ds Fraser, Tom Blakeman. Chronic kidney disease: further development of the concept and classification. Pragmat Obs Res. 2007;11(4):7-17.

8. Ernesto L Schiffrin 1, Mark L Lipman, Johannes F E Mann. Chronic kidney disease: effects on the cardiovascular system. Circulation. 2007;116(1):85-97.

9. National Kidney Foundation. K/DOQI clinical practice guidelines for chronic kidney disease: evaluation, classification, and stratification / National Kidney Foundation. Am J Kidney Dis. 2002;39(2 Suppl 1):1266.

10. Ye V Makarov, AN Maksudova, ID Shakirov. The state of the cardiovascular system in patients undergoing program hemodialysis. Practical medicine. 2015;7(7):124-129. 